Institute of Health Informatics, University College London, London

ami.banerjee@ucl.ac.uk Twitter @amibanerjee1 Cite this as: BMJ 2021;375:n2736 http://dx.doi.org/10.1136/bmj.n2736 Published: 9 November 2021

\section{Long covid: new wine in need of new bottles}

\section{Finding solutions to long covid will require new ways of thinking across clinical services and research, says Amitava Banerjee}

\section{Amitava Banerjee professor of clinical data science and honorary consultant cardiologist}

William Osler famously said, "Listen to the patient, he is telling you the diagnosis." For long covid, patients have not only told us the diagnosis since April 2020, but also gave a clear steer as to how we should proceed with this new disease: "recognition, research, and rehabilitation." ${ }^{1}$ We have made mixed progress across these areas.

It is not hard to see why we have not always been successful in responding to long covid. From HIV/AIDS to Ebola, emerging disease threats with coordinated international responses are usually acute and infectious. A new chronic condition, albeit as a post-acute complication of SARS-CoV-2 infection, requires new ways of thinking across clinical, public health, policy, and academic disciplines. Whether it be the World Health Organization (WHO), governments, or research funders, emergency preparedness has tended to exclude chronic and post-infectious disease management, but long covid must change that.

Recognition of a new disease requires it to be classifiable and recordable, facilitating monitoring, research, and treatment. SNOMED CT codes for long covid were developed and released in the UK in November 2020, ${ }^{2}$ followed by ICD-10 codes and a WHO case definition in June and October of 2021. ${ }^{34}$ However, the uptake of these codes is still low in the $\mathrm{UK}^{2}$ and worldwide, leading to a systematic underestimation of the disease burden of long covid in routine care, despite high estimated prevalence from self-reported surveys (e.g. 1.7\% of the UK population as of 5 September 2021). ${ }^{5}$

As the UK enters a second winter of the pandemic, long covid is still Cinderella at the ball of covid-19 discourse. The vast majority of policy documents, political discussions, research, and public health interventions over the past two years have tackled the acute impact of SARS-CoV-2, with limited consideration of its long term sequelae. Even when we've faced Groundhog Day moments of rising cases and hospitalisations, the argument for avoiding and managing long covid has been neglected by policy makers-whether it be as a reason to suppress infection, increase vaccination rates, protect the NHS, or avoid economic downturn. We know the inevitability of countries worst affected by the pandemic experiencing a chronic burden of morbidity, yet there is still no policy recognition of the scale of threat from long covid.

The good news is that research is now being funded at scale. The National Institute for Health Research (NIHR) has so far funded two rounds of research focused on long covid, awarding £18.6 million for four studies in February 2021, ${ }^{6}$ and £19.6 million for
15 studies in July 2021. ${ }^{7}$ For example, in the STIMULATE-ICP study (Symptoms, Trajectory, Inequalities and Management: Understanding Long-COVID to Address and Transform Existing Integrated Care Pathways), in which I am a lead investigator, we will be conducting the largest trial on long covid to date. We will be recruiting more than 4500 people to test the effectiveness of existing drugs by measuring the effect of three months' treatment on symptoms, mental health, and other outcomes such as returning to work. We will also assess the use of MRI scans to help diagnose potential organ damage, as well as digitally enhanced rehabilitation to track patients' symptoms and recovery.

In the US, the National Institutes of Health announced in September 2021 that US\$470 million would be dedicated to long covid research projects. ${ }^{8}$ Across countries, and even across the NIHR portfolio of projects, from epidemiology and health economics to treatment interventions and core outcome datasets, clinically relevant research is happening at pace.

Rehabilitation for a new chronic disease such as long covid relies on the right health system responses and the right research. Yet some commentators have raised concerns that not enough research is being done as services are implemented. ${ }^{9}$ The ITU management of acute covid occurred simultaneously with platform trials such as RECOVERY - an approach that worked well. Similarly, long covid research, including trials, must happen at the same time as dedicated services are rolled out across countries, to support integrated care for the hundreds of thousands of affected individuals already seeking investigation and treatment.

Health professionals and academics may see boundaries between clinical service versus research, or between primary and secondary care, but patients do not, and our solutions shouldn't either. The acknowledgment of the limitations of our current knowledge needs to be combined with a willingness to move in a changing landscape, whether that means shifting definitions of hospitalised versus non-hospitalised long covid, using rapidly emerging preclinical data to understand and test underlying mechanisms, or testing treatments in general patient populations in order to establish disease subtypes. Where academic expertise is not yet developed or established, funding and advisory panels need to incorporate greater frontline clinical and lived experience.

When it comes to long covid, traditional models of care and research need to move pragmatically, adaptively, iteratively, and rapidly, considering dissemination and implementation in 
parallel $^{10}$-otherwise, we risk finding the answers after the train has left the station.

Competing interests: AB has received grant funding from AstraZeneca, NIHR, UKRI, European Union, and the British Medical Association. He is PI of the STIMULATE-ICP study(https://www.stimulate-

icp.org/). He is also a trustee of the South Asian Health Foundation.

1 Carson GLong Covid Forum Group. Research priorities for Long Covid: refined through an international multi-stakeholder forum. BMC Med 2021;19:84.

doi: 10.1186/s12916-021-01947-0. pmid: 33785027

2 Walker AJ, MacKenna B, Inglesby P, etalThe OpenSAFELY Collaborative). Clinical coding of long COVID in English primary care: a federated analysis of 58 million patient records in situ using OpenSAFELY. BrJ Gen Pract 2021;11:e806-14. doi: 10.3399/BJGP.2021.0301. pmid: 34340970

3 World Health Organization. A clinical case definition of post COVID-19 condition by a Delphi consensus. 6 October 2021. https://www.who.int/publications///item/WHO-2019-nCoVPost_COVID-19_condition-Clinical_case_definition-2021.1

4 World Health Organization. International Classification of Diseases (ICD): Emergency use ICD codes for COVID-19 disease outbreak. https://www.who.int/standards/classifications/classificationof-diseases/emergency-use-icd-codes-for-covid-19-disease-outbreak

5 Office for National Statistics. Prevalence of ongoing symptoms following coronavirus (COVID-19) infection in the UK: 7 October 2021. https://www.ons.gov.uk/peoplepopulationandcommunity/healthandsocialcare/conditionsanddiseases/bulletins/prevalenceofongoingsymptomsfollowingcoronaviruscovid19infectionintheuk/7october2021

6 National Institute for Health Research. f18.5 million awarded to new research projects to understand and treat long COVID. 18 February 2021. https://www.nihr.ac.uk/news/185-millionawarded-to-new-research-projects-to-understand-and-treat-long-covid/26895

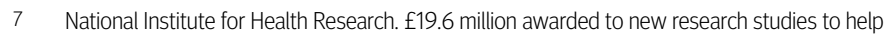
diagnose and treat long COVID. 18 July 2021. https://www.nihr.ac.uk/news/196-million-awardedto-new-research-studies-to-help-diagnose-and-treat-long-covid/28205

8 Winter L. NIH Grants $\$ 470$ Million for Study of Long COVID. The Scientist. https://www.the-scientist.com/news-opinion/nih-grants-470-million-for-study-of-long-covid-69192

9 McCartney M, Byng R. Long covid clinics should be run as research hubs. BMJ2021;374:n1996. doi: 10.1136/bmi.n1996. pmid: 34417203

10 Yousefi Nooraie R, Shelton RC, Fiscella K, Kwan BM, McMahon JM. The pragmatic, rapid, and iterative dissemination and implementation (PRIDI) cycle: adapting to the dynamic nature of public health emergencies (and beyond). Health Res Policy Syst 2021;19:110.

doi: 10.1186/s12961-021-00764-4. pmid: 34348732 